\title{
TAXONOMY OF OPTIMIZATION APPROACHES OF RESOURCE BROKERS IN DATA GRIDS
}

\author{
Rafah M. Almuttairi \\ University of Babylon, Babylon, Iraq
}

\begin{abstract}
A novel taxonomy of replica selection techniques is proposed. We studied some data grid approaches where the selection strategies of data management is different. The aim of the study is to determine the common concepts and observe their performance and to compare their performance with our strategy.
\end{abstract}

\section{KEYWORDS}

Data grid Optimizer; Taxonomy ; Selection technique; Broker.

\section{INTRODUCTION}

In Data Grid Architecture, the optimizer or resource broker is a tool used where the decision should be taken. That means optimizer is needed whenever there is a need to determine when or how to acquire the services and/or resources for components in higher level. There are many replica optimizers which used different replica selection proposed and developed by research groups and companies.

In Data Grid Job, file access pattern varies between jobs as shown in Figure 1, therefore there are different directions that have been followed by researchers to minimize total jobs execution time. The first direction that has been followed by researcher is focused on minimizing the time that consumes in the selecting set of best replica sites, so, they proposed different selection strategies used by Replica Optimization Service to enhance selection process [2]. The objective of the other direction is to exploit rate differences among links of various computing data grid site and replica data grid providers and also to divide the requested files into multiple blocks sizes for improving the efficiency of transferring files in grids [12]. When the files dividing into multiple parts, then co-allocation architecture is used to transfer the files in parallel from multiple replica provider sites. 

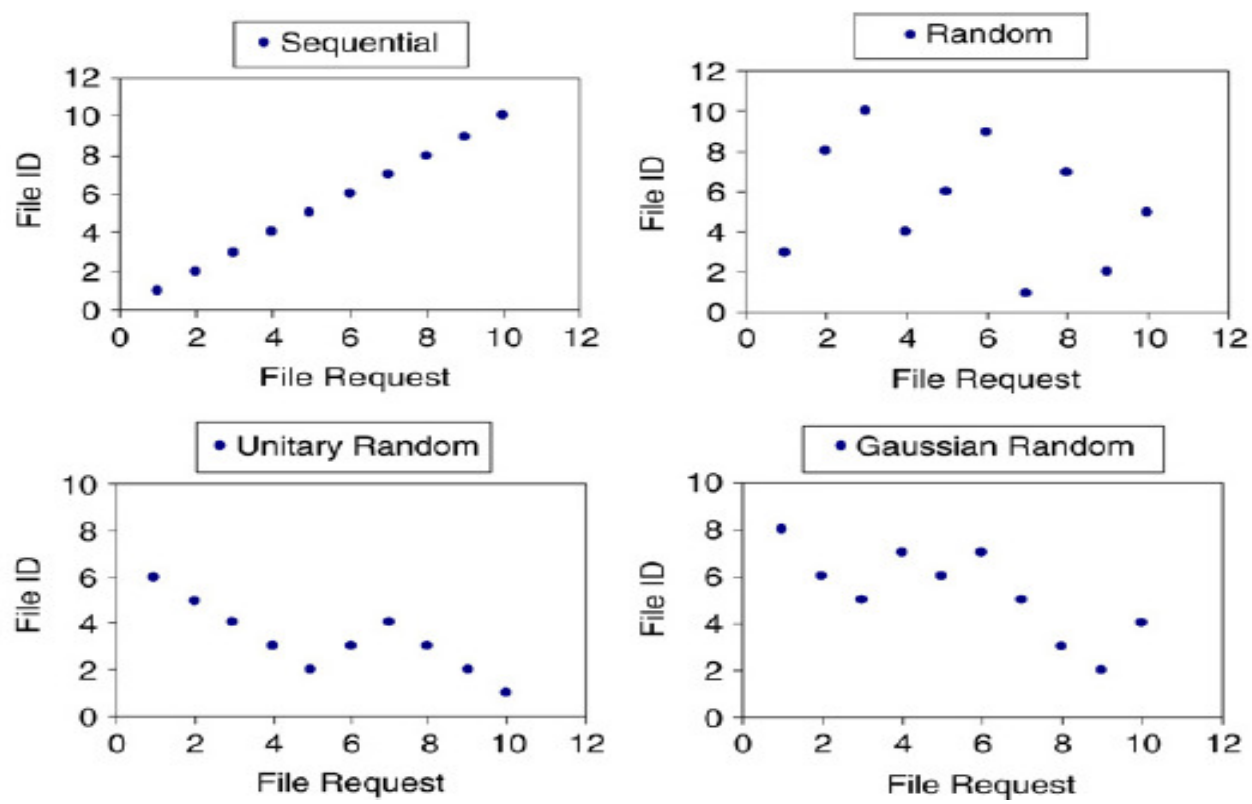

Figure 1: Various file access patterns [3]

\section{History Of Selection STRATEgies}

In this section a brief history of the most known selection strategies that are used in Data Grid Brokers.

R. M. Almuttairi et al. [1]. This is our proposed work, it is a replica selection Broker that can be used to reduce the cost of total time of Data Grid job. In this work, a set of uncongested network links of replica providers are selected. The selected set of providers have a close match to the customer's requirements.

J. Gwertzman with M. Seltzer [27] and J. Guyton with M. Schwartz [30] In this work they proposed a new selection strategy which is binding a computing site to the nearest replica provider site. Both of static metric are used in this method, first, the distances counted by number of Hops [31] which is called "topological distance" and the second which is called "geographical distance" in miles [31].

The experimental results in $[15,25]$ show, that using such static metrics (topological distance, geographical distance ), for prediction process won't give an accurate results for the total response time of customer/client requests. The weakness of any method use a static metric such as geographical and topological distance network metrics, is because these metrics ignore the reality of dynamic condition which effect on the network link's.

R. Kavitha, and I. Foster [28], In this work they proposed another selection strategy that is use the traditional catalog of replicas. In their work, for each new replica request the service of replica location called "Replica Location Service, RLS" is queried. RSL is used to get the physical addresses of replica provider sites. After getting all providers that have same copy of the requested files, another grid service should be queried to probe the links of network that connect computing site with all replica provider sites. Network service here is using the "HopCount method". After that the list of providers with number of Hops between each one and computing 
site will be ready to be used to select best replica provider site. The best replica provider is the one who has the least number of Hops. This method has some drawbacks. One of these drawbacks is that it depends on the number of hops, the least number of hops will not guarantee that the selected provider's links is the best. As number of hops might not give the real and actual condition of the network such as the capacity of bandwidths and the latency of links.

Sudharshan et al. $[22,5,20]$ In this work the authors proposed another new selection strategy for replica management in Data Grids. They used history of transferring requests. The all previous file transfer information is used to predict the best replica provider site that have exact copy of the requested file. The history file save some information of previous data grid jobs, such as: file size, the bandwidth of the link between the selected provider and computing element and total transfer time spent to move all data between two sites. The information in the history file can be used by many prediction techniques. In this work these information of history file is used to train and test regression prediction model. After that, the trained model is used to predict the actual total time of transferring the requested file. Another thing they tried to show in their model was about getting better prediction if the model used history of data requests of various sources which help to get better predictions than getting requested data from one source. Accurate results are achieved when they used the trained data from all of these three sources: file size, network data streams, and past data grid transfer information.

Several heuristic data replication schemes have been proposed in the literature $[33,17,18,34,35$, 36]. A dynamic replication scheme was proposed based on a variety Vickery auction for an economy based replication solution which is discussed in [17].

F. Corina with M. Mesaac [23] and Ceryen with M. Kevin [17] used different algorithms such as greedy, random, partitioned and weight algorithms in the selection engine.

Rashedur et al. [6] This work is about another replica provider selection technique. Here, algorithm of K-Nearest Neighbor rule is used to select the best replica provider. Using information of replica provider sites which is gathered locally. This method selects the best replica provider to get file from by considering all previous file transfer logs which indicating the history of the transferring the requested file and those similar. This technique is not efficient as it depends on the access patterns as shown in Figure 1. Therefore, the misclassification in common to be occurred especially in the Gaussian random access pattern when the accuracy is the lowest. There is another drawback in this technique, that all previous file requests should be saved to be used in the next selection process. This will increase the wasted time which is used to search in the large Database history file and finally the selected replica site might or might not be requested one.

H. Lin, et al [2] In this work, they have proposed a new data replication policy. The new proposed policy is to improve replication the common used files (replicas). It is been implemented in data resource management system in Data Grid. This has improved the replication when a variable pricing scheme are used, that was based on the expected actions of a rational agent compared to a fixed pricing scheme. Also, this work enhance cost and speed of file transferring using the proposed Broker, i.e. the performance of the data grid network traffic is improved.

Rashedur et al. [6] A predictive technique is used in this work to estimate the total file transfer time between replica provider sites. In Neural Network (NN) strategy, the predicted time of file transfer could be used as an estimation time to select best replica provider site among different replica provider sites. Results of simulation, shows that predictive used technique is more accurately than the other predictive technique such as multi-regression model. However, multiregression technique has been used before of Neural Network technique [22, 20, 5]. 
Nevertheless, Neural Network technique is not stable, it sometimes gives right selection decision, the reason is because the copy of the replica may not be available in the predicted provider site that is a common occurrence in grid, when this problem happens so, the Traditional selection Model has to be used instead of Neural Network technique.

A. Jaradat et al. [9] this work is to select replica provider using K-means algorithm , the concept of clustering algorithm is used to create a best solution. In this work, the selected replica site does not have the shortest file transfer time, but, the selected replica site should has accepted values of affected parameters such as: the level of file security, availability and file transfer time. This method has a drawback that is related to use K-means algorithm. The selection process is being trapped in local clustering centroids. However, at a given state of the grid The problem is about finding an optimal replica allocation, that means, replica provider that has minimum transfer cost for a given read-write pattern [29].

Vazkhudai et al. [21, 5] show that disk I/O effects on the transfer time in Grid environment so, all these criterion can be used during the selection process.

\subsection{Drawbacks of the selection Methods}

Here brief drawbacks of previous selection strategies are listed:

1. History file is used in K-Nearest Neighbor rule $(\mathrm{KNN})$ method, it does not react the recent information; it is outdated information

2. Bandwidth alone and Hop counts alone are used in Traditional Method (TM), Neural Network (NN) and KNN methods, each might not describe the real network condition.

\subsection{Taxonomy of Replica Selection Strategies}

Figure. 2. explains the taxonomy of replica selection techniques which are used in the management system of replica selection process in Data Grids.

Replica Optimization Service ROS is an internal service component of the Replica Management Services used as a selection service [2]. It is classified into three types which are:

* Scheduling Optimization: This term is used to name the long-term scheduling strategy, replica optimization strategy is used to allocate the data grid job to suitable location grid's site and also has a suitable computational capabilities.

* Short-term Optimization: This term is used to name the type of selection strategies where the decision of choosing best replica site depending on network conditions such as availability of network bandwidth.

* Long-term Optimization: This term is used replica optimization strategy is used to create and delete replica on the Grid according to its predictions of file usage across the grid. Here, the strategy decides which file should be retained as a replica and which one should be deleted[26].

As we mentioned, selection problem has been investigated by many researchers. The research work is divided into two aspects: first was related to enhancement of the replica selection strategy. Second aspect is related to enhancing the file transfer strategy [2].

The objective of the first aspect is to enhance selecting process by new methods which select set of replica servers instead of one replica provider site [1], whereas the second aspect aims to 
improve the efficiency of transferring the requested files between the selected computing sites and replica provider sites [12].

To optimize job in such a way that satisfy the requirement of the Data Grid's user/application, replicas selection strategies provide the best single replica site or the best set of replicas $(M$ : number of replica sites). However, Replica Selection services are classified according to how many providers could be selected simultaneously while applying replica selection strategy. There are two types which are:

* A Single replica provider: To determine the best replica provider site among all available replica sites.

* Multiple replica providers: To determine multiple replica provider sites among all available replica provider sites. The Link Analyzes by Association Rules is used to find the hidden relationships of network links of replica providers [16].

In both of above methods, the total execution time and the total cost (price) of obtaining a replica (file) are the most important factors required by a user/application. However, the requirements of any data grid user/application can be described through:

* Minimizing (reducing) Cost: Here, the selection strategy selects the cheapest replica to be the best replica site using the answer from auction protocol [1]

* Minimizing (reducing) Time: Here, the selection strategy attempts to reduce the total job execution time in different ways such as minimizing the look up time, and/or searching time and/or transmission time.

* Minimizing both: Here, the selection strategy reduces cost and time such that the answer from selection strategy is used in the economy-based data replication Broker [2]

According to reduce the total execution time, the selection strategies of previous works are classified into three types according to the time period they wanted to minimize. The previous strategies had aimed to:

1. Reducing the lookup time: Usually most of selection approaches, minimize total execution time by minimizing time of replicas selection process. This can be done by reducing catalog lookup time and probing network links time. It is done either by using a classification technique such as K-Nearest Neighbor rules [6] or predictive technique like Neural Network techniques [3] or Genetic algorithm technique [4]. All these methods attempt to avoid lookup time during predicting the total transferring time. All tried to enhance the traditional models which used the catalog look up time

2. Reducing data transmission time: This is to reduce the total time of file transfer, various selection methods were used such as, selection of the highest bandwidth among replicas or the shortest round trip time link, or the least number of hops or the shortest geographical distance. Recently association rules mining is also proposed by us [19]

3. Reducing the searching time: In this process to get list of providers, the catalog must be looked up. That means, the catalog lookup time cannot be ignored. The searching time is the time that is consumed to find the replica provider who has the closest match to the user/application requirements. This cost of time can be minimized by various selection strategies as researchers worked on, which are:

a) Random selection: The best replica is chosen randomly [7]

b) Round Robin: Round robin method is used for the selection process [8]

c) Sequential searching method: Sequential Method is used for the selection process [9]. These methods can be used in case of equality of the replica's 
International Journal of Computer Science \& Information Technology (IJCSIT) Vol 7, No 2, April 2015

attributes where Euclidean distance equation can be sequentially used to find the shortest distance between the user requirement and the list of replicas.

d) Classification Modeling: Used to classify the replicas using the history information to select the best one such as K-Nearest Neighbor rules [6].

e) Predictive Modeling: Used to predict the best replica depending on the history sa78i8ewsaz information such as regression [5] or Neural Network techniques [6].

f) Database Segmentation/ Clustering: In this method the replicas are grouped into different clusters, the replicas within a cluster have similar characteristics so the distance between two replicas in a cluster is smaller than between two replicas belonging to different clusters. These approaches used different clustering methods which are:
1. K-means algorithm [9]
2. Genetic algorithm [4]
3. Grey-based Rough Set Theory (GRST) [10]

In the selection techniques of GRST the following concepts is used:

1. Lower and Upper approximations [10]

2. Reduct [11] 
International Journal of Computer Science \& Information Technology (IJCSIT) Vol 7, No 2, April 2015

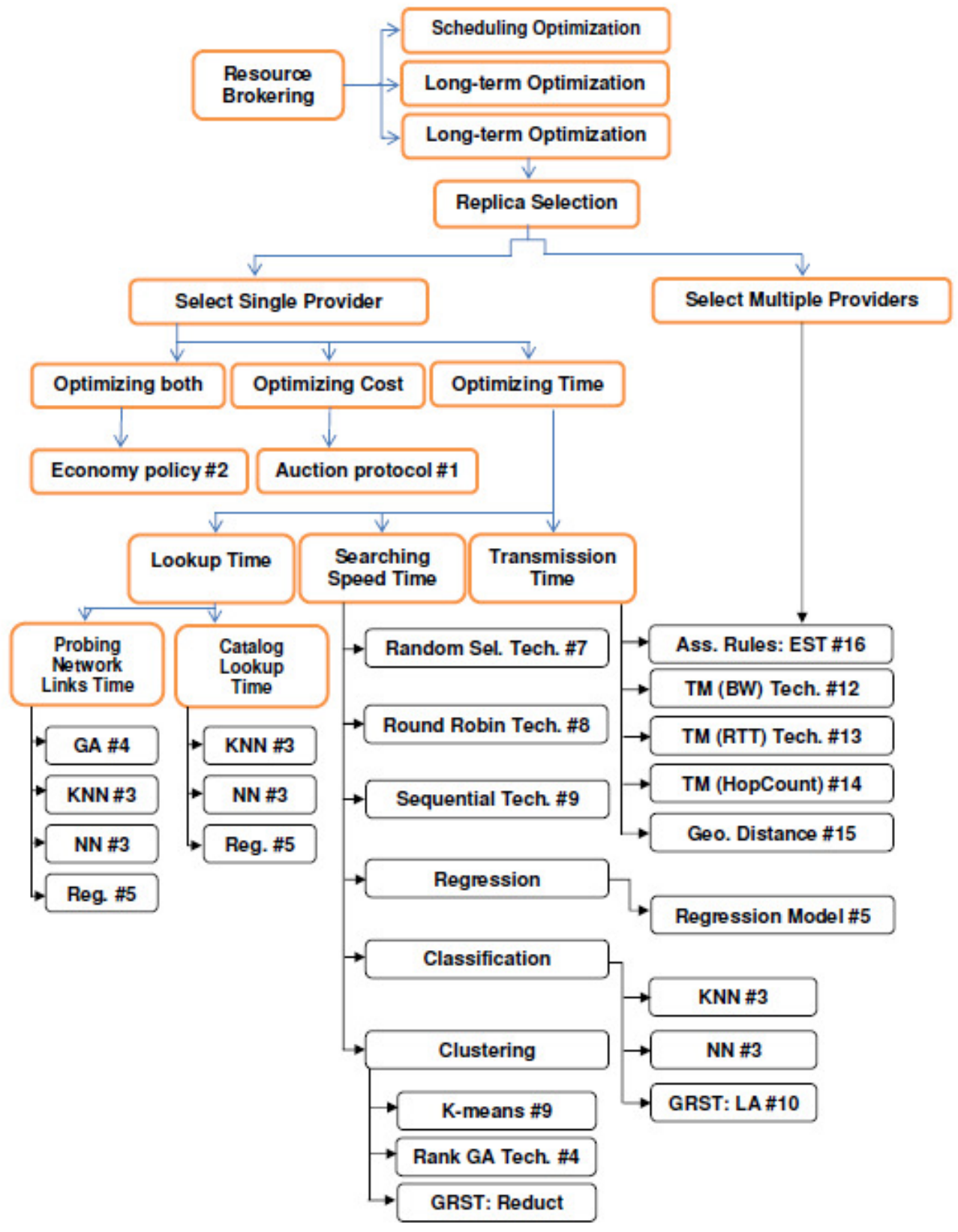

Figure 2: A Taxonomy of Replica Selection Techniques

\section{Comparison of Selection Techniques}

This section is to compare among several methods which have been used in replica selection techniques including ours. The previous selection techniques that has been used to compare our technique with is discussed in Taxonomy section and shown in Figure 3 below.

To compare these methods, the OptrSim[37]simulator is used with code of C++ which is responsible of calculating total transfer time using five different techniques. 
As it is shown in Figure [3], The total transferring time is varies among the strategies, let us analyze that when they all techniques are used to transfer the requested file which is named as $F 1$. $F 1$ has been stored in different replica sites, list of replica providers can be updated and got it using Dynamic Grid Resource Information. Network Information of links connected replica providers with computing site can be updated and got is using Network Monitoring Service. Using these information, we execute all five strategies (TM (HopCount), TM(BW), KNN with both probabilities right and wrong classification, NNM with both probabilities right and wrong prediction and last our proposed strategy EST.

Results of total file transferring time value varies with different Network links conditions during file transfer. The drawbacks that is mentioned in section $\mathbf{3}$ of this article were the main reasons for the results shown below. The best selection strategy in the most case was EST as it is taking care of recent network condition history and selects set of replica providers to share transferring parts of requested files.

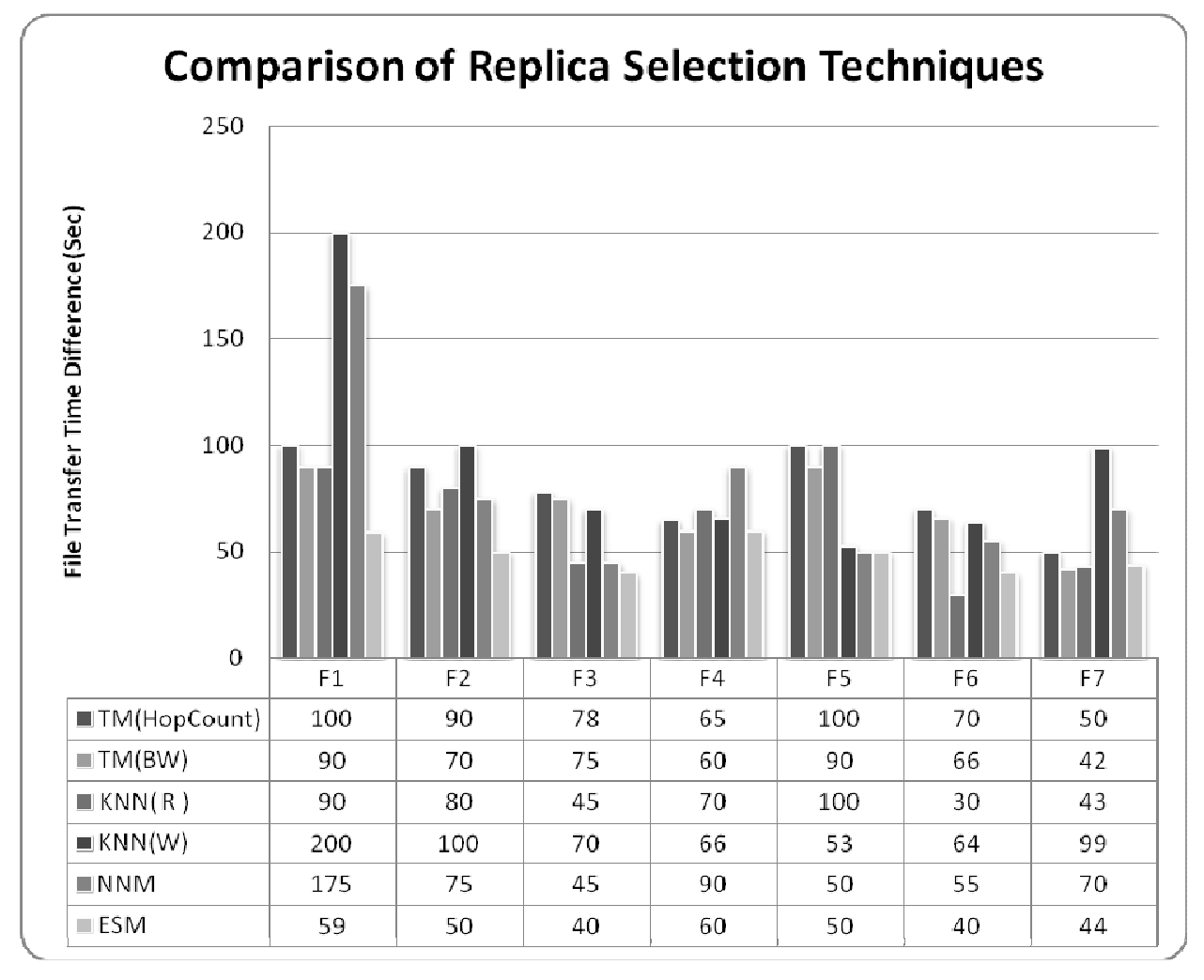

Figure 3: Comparison of Replica Selection Techniques

\section{Summary}

This article arranged as follow, first an introduction to replica selection Brokers in Data Grids is briefly explained. Then a history of selection strategies is clearly present. After that our proposed taxonomy of replica selection strategies is given. And then, a comparison of six different techniques including ours has been cleared. 
International Journal of Computer Science \& Information Technology (IJCSIT) Vol 7, No 2, April 2015

\section{REFERENCES}

[1] R.M. Almuttairi, R. Wankar, A. Negi, C.R. Rao, A. Agrawal, R. Buyya, A two phased service oriented broker for replica selection in data grids (2SOB), Future Generation Computer Systems (2013), doi:10.1016/j.future.2012.09.007. ISSN 0167-739X.

[2] H. Lin, J. Abawajy, and R. Buyya. Economy-based data replication broker. In Proceedings of the Second IEEE International Conference on e-Science and Grid Computing, e-Science '06, page 90, 2006.

[3] R. M. Rahman, R. Alhajj, and K. Barker. Replica selection strategies in data grid. In Journal of Parallel Distributing and Computing, volume 68, pages 1561-1574, 2008

[4] O. Aljadaan, W. Abdulal, M.A. Hameed, and A. Jabas. Enhancing data selection using genetic algorithm. In Proceedings of the 2010 International Conference on Computational Intelligence and Communication Networks (CICN), pages 434-439, 2010.

[5] S. Vazhkudai and J. Schopf. Using regression techniques to predict large data transfers. In International Journal of High Performance Computing Applications (IJHPCA), Baltimore, August 2003.

[6] R. M. Rahman, K. Barker, and R. Alhajj. Predicting the performance of gridftp transfers. In Proceedings of the International Parallel and Distributed Processing Symposium (IPDPS'02) 2004.

[7] C. Tan and K. Mills. Performance characterization of decentralized algorithms for replica selection in distributed object systems. In Proceedings of the 5th International Workshop on Software and Performance (WOSP 2005), pages 257- 262, 2005.

[8] M. Lei, S.V. Vrbsky, and Q. Zijie. Online grid replication optimizers to improve system reliability. In Proceedings of the IEEE International Parallel and Distributed Processing Symposium, 2007 (IPDPS 2007), pages 1-8, 2007.

[9] A. Jaradat, R. Salleh, and A. Abid. Imitating k-means to enhance data selection. In Journal of Applied Sciences 2009, volume 19, pages 3569-3574, 2009.

[10] R. M. Almuttairi, R. Wankar, A. Negi, and C. R. Rao. Smart replica selection for data grids using rough set approximations. In Proceedings of the The International Conference on Computational Intelligence and Communication Networks (CICN2010), pages 466-471, Los Alamitos, CA, USA, 2010. IEEE Computer Society.

[11] R. M. Almuttairi, R. Wankar, A. Negi, and C. R. Rao. Rough set clustering approach to replica selection in data grids. In Proceedings of the 10th International Conference on Intelligent Systems Design and Applications (ISDA), pages 1195-1200, Egypt, 2010.

[12] C-T. Yang, I-H. Yang, C-H. Chen, and S-Y. Wang. Implementation of a dynamic adjustment mechanism with efficient replica selection in data grid environments. In Proceedings of the 2006 ACM symposium on Applied computing, SAC '06, pages 797-804, New York, NY, USA, 2006. ACM.

[13] S. Vazhkudai, S. Tuecke, and I. T. Foster. Replica selection in the globus data grid. In Proceedings of the 3rd IEEE/ACM International Symposium on Cluster Computing and the Grid, 2003. Proceedings, CCGrid 2001, pages 106-113, 2001.

[14] Cisco Distributed Director. Cisco distributed director http://www.cisco.com/warp/public/cc/pd/cxsr/dd/index.shtml.

[15] M. Sayal, Y. Breitbart, P. Scheuermann, and R. Vingralek. Selection algorithms for replicated web servers. In Sigmetrics Performance Evaluation Review, volume 26, pages 44-50, 1998.

[16] R. M. Almuttairi, R. Wankar, A. Negi, and C. R. Rao. Intelligent replica selection strategy for data grid. In Proceedings of the 2010 International Conference on Grid Computing and Applications (GCA'10), pages 95-101, 2010.

[17] W. H. Bell, D.G. Cameron, R. Carvajal-Schia_no, A.P. Millar, K. Stockinger, and F. Zini. Evaluation of an economy-based_le replication strategy for a data grid. In Proceedings of the $3^{\text {rd }}$ IEEE/ACM International Symposium on Cluster Computing and the Grid, 2003. Proceedings, CCGrid 2003, pages 661- 668, May 2003.

[18] M. Carman, F. Zini, L. Sera_ni, and K. Stockinger. Towards an economy \{ based optimization of file access and replication on a data grid. In Proceedings in Workshop on Agent based Cluster and Grid Computing at 2nd International Symposium on Cluster Computing and the Grid (CCGrid2002), pages 286- 297, May 2002. 
[19] R. M. Almuttairi, R. Wankar, A. Negi, C. R. Rao, and M. S. Almahna. New replica selection technique for binding replica sites in data grids. In Proceedings of 1st International Conference on Energy, Power and Control (EPC-IQ), pages 187-194, Dec. 2010.

[20] S. Vazhkudai, S. Tuecke, and I. T. Foster. Replica selection in the globus data grid. 2002.

[21] S. Vazhkudai and J. Schopf. Using disk throughput data. In Proceedings of the 3rd International Workshop on Grid Computing in Predictions of End-to-End Grid Transfers, Baltimore, 2002.

[22] I. Foster, S. Vazhkudai, and J. Schopf. Predicting the performance of wide-area data transfers. In Proceedings of the 2008 ACM/IEEE conference on Supercomputing (SC 2008), pages 286-297, Nov. 2008.

[23] C. Ferdean and M. Makpangou. A scalable replica selection strategy based on exible contracts. In Proceedings of the Third IEEE Workshop on Internet Applications, WIAPP 2003, pages 95-99, June 2003.

[24] Open grid services architecture (ogsa). http://www.globus.org/ogsa.

[25] E. W. Zegura, M.H. Ammar, F. Zongming, and S. Bhattacharjee. Application-layer any casting: a server selection architecture and use in a replicated web service. In Proceedings of the Transactions on Networking, IEEE/ACM, volume 8, pages 455-466, Augest 2000.

[26] Wp2, http://edg-wp2.web.cern.ch/edg-wp2/optimization/ros.html.

[27] J. S. Gwertzman and M. Seltzer. The case for geographical push-caching. In Proceedings of the 5th Workshop on Hot Topics in Operating Systems, 1995. (HotOS-V), pages 51-55, May 1995.

[28] R. Kavitha and I. Foster. Design and evaluation of replication strategies for a high performance data grid. In Proceedings of the CHEP'01, pages 106-118, Beijing, 2001.

[29] O. Wolfson and S. Jajodia. An algorithm for dynamic data allocation in distributed systems volume 53, pages 113\{119, Amsterdam, The Netherlands, The Netherlands, January 1995 .Elsevier NorthHolland, Inc.

[30] J. D. Guyton and M. F. Schwartz. Locating nearby copies of replicated internet servers. In Proceedings of the conference on Applications, technologies, architectures, and protocols for computer communication, SIGCOMM '95, pages 288\{298, New York, NY, USA, 1995. ACM.

[31] Cisco distributed director. http://www.cisco.com/warp/public/cc/pd/cxsr/dd/index.shtml.

[32] U. Kant and D. Grosu. Double auction protocols for resource allocation in grids. In Proceedings of the International Conference on Information Technology: Coding and Computing (ITCC'05, volume 1, pages 366-371, Washington, DC, USA, 2005. IEEE Computer Society.

[33] K. Holtman. Object level replication for physics. In Proceedings of the 4th Annual Globus Retreat, Pittsburgh, July, 2000.

[34] K. Ranganathan and I. Foster. Identifying dynamic replication strategies for a high performance data grid. In Proceedings of the International Grid Computing Workshop, pages 286\{297, Nov. 2001.

[35] T. Loukopoulos and I. Ahmad. Static and adaptive data replication algorithms for fast information access in large distributed systems, icdcs. In Proceedings of the 20th International Conference on Distributed Computing Systems (ICDCS 2000), 2000.

[36] J. J. Abawajy. Placement of file replicas in data grid environments. pages 66-73, 2004.

[37] Lamehamedi, H.; Shentu, Z.; Szymanski, B.; Deelman, E., "Simulation of dynamic data replication strategies in Data Grids," Parallel and Distributed Processing Symposium, 2003. Proceedings. International, vol., no., pp.10 pp.,, 22-26 April 2003.

\section{Author}

Rafah M. Almuttairi is currently a faculty member at the University of Babylon, Babylon, Iraq. She has completed her Ph.D. research in University of Hyderabad, India in April, 2012. She received her Master's Degree in Computer Science from Baghdad University, Iraq in October, 2003. She received her Bachelor's Degree in Computer Science and Bachelor Degree in Physics from University of Babylon, Iraq in October, 2001 and October 1994 respectively. She has authored several international conference papers in the area of grid computing. Her current research interest is in the area of data grid architecture and fuzzy decision making for grid resources and replica selection.

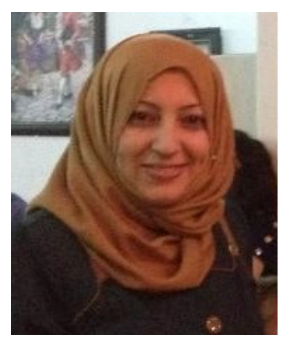

